\section{Case Reports in Ophthalmology}

Case Rep Ophthalmol 2021;12:25-31

DOI: $10.1159 / 000510558$

Published online: January 7, 2021
(C) 2021 The Author(s)

Published by S. Karger AG, Basel www.karger.com/cop

This article is licensed under the Creative Commons Attribution-NonCommercial 4.0 International License (CC BY-NC) (http://www.karger.com/Services/OpenAccessLicense). Usage and distribution for commercial purposes requires written permission.

\title{
Paracentral Acute Middle Maculopathy following Surgically Induced Branch Retinal Artery Occlusion During Vitrectomy
}

\author{
Panagiotis Stavrakas $^{a}$ Efthymios Karmiris $^{b}$ Paris Tranos $^{c}$ \\ Konstantinos Ananikas ${ }^{d} \quad$ Christos Tooulias $^{d} \quad$ Alexandros Rouvas ${ }^{d}$ \\ Angeliki Androu ${ }^{\mathrm{d}}$ \\ aDepartment of Ophthalmology, University of Patras Medical School, Patras, Greece; \\ ${ }^{b}$ Department of Ophthalmology, Hellenic Air Force General Hospital, Athens, Greece; \\ "Ophthalmica" Institute of Ophthalmology, Thessaloniki, Greece; d2nd Department of \\ Ophthalmology, University of Athens Medical School, Attikon University General Hospital, \\ Athens, Greece
}

\section{Keywords}

Paracentral acute middle maculopathy · Capillary ischaemia · Pars plana vitrectomy

\begin{abstract}
Paracentral acute middle maculopathy (PAMM) is a spectral-domain OCT finding of a thickened hyperreflective band at the level of the intermediate layers of the inner retina, attributed to the acute phase of intermediate capillary ischemia. The purpose of this paper is to report a case of PAMM deriving from a surgically induced branch retinal artery occlusion (BRAO) during vitrectomy for vitreous hemorrhage. A 70-year-old female referred to the posterior segment office with a 1-month history of visual loss in her right eye. Best-corrected visual acuity (BCVA) was "counting-fingers" (CF) due to a dense vitreous hemorrhage. She underwent a standard 23-gauge vitrectomy with a relatively close to the optic disc endodiathermy application to an avulsed vessel. Follow-up examination revealed a well-demarcated retinal whitening involving the inferior macula. SD OCT showed a surgically induced branch retinal artery occlusion exhibiting both a cotton wool spot and a PAMM lesion. Our case affords an insight into those
\end{abstract}




\section{Case Reports in Ophthalmology}

pathological processes involved with PAMM, providing angiographic evidence of the retinal ischemic changes responsible for its development, by angiographically proving that branch retinal artery occlusion leads to a prompt formation of both a cotton wool spot and a PAMM lesion. Our aim is to raise awareness of this potential complication of vitrectomy surgery, especially when involving multiple traumatic manipulations onto the retinal surface.

(C) 2021 The Author(s)

Published by S. Karger AG, Basel

\section{Introduction}

Paracentral acute middle maculopathy (PAMM) is a novel descriptive term introduced by Sarraf et al. in 2013 [1] referring to a spectral-domain optical coherence tomography (SDOCT) finding of a thickened hyperreflective band at the level of the deeper layers of the inner retina [2]. PAMM has been attributed to the acute phase of deep capillary ischemia, referring to ischemia of intermediate and deep capillary plexuses [2]. Since then, PAMM has been associated with various retinal vascular diseases, including retinal artery and central retinal vein occlusion, diabetic retinopathy, sickle cell retinopathy, Purtscher's retinopathy, inflammatory occlusive retinal vasculitis and hypertensive retinopathy [3], all sharing a similar pathophysiological background.

Similarly, multiple reports in the published literature have focused on the incidence of PAMM lesions secondary to intraocular surgery [4-6]. Creese et al. [5] demonstrated PAMM incidence following uncomplicated phaco-emulsification cataract surgery under local anesthesia using retrobulbar, peribulbar or sub-tenon's techniques, causing severe irreversible vision loss in a case series report. They speculated that pre-existing compromised retinal perfusion, due to medical history of vasculopathy and/or blood dyscrasias, may render some eyes more susceptible to transient compression or spasm of the central retinal artery [5]. Additionally, Nakashima et al. [6] reported a percentage as high as 3.8\% of PAMM following vitrectomy for proliferative diabetic retinopathy (PDR), also focusing on underlying retinal vascular dysfunction, leading to local perfusion insufficiencies in diabetic patients.

The purpose of this paper is to report a case of PAMM in combination with ischemic manifestations deriving from the superficial capillary plexus following a surgically induced branch retinal artery occlusion during vitrectomy for vitreous hemorrhage.

\section{Case Presentation}

A 70-year-old female with past medical history of arterial hypertension presented with a 1-month history of visual loss in her right eye. On evaluation, best-corrected visual acuity (BCVA) was "counting-fingers" (CF) and 20/20 in the right and left eye, respectively. Examination revealed a dense vitreous hemorrhage obscuring fundus view in her right eye, not suggestive of retinal detachment, based on b-scan prompt examination. Fundoscopic examination of the left eye was totally unremarkable. Intraocular pressure was $16 \mathrm{~mm} \mathrm{Hg}$ in both eyes. On the basis of these findings, a both diagnostic and therapeutic right eye surgical intervention under local anesthesia was scheduled. She underwent a standard 23-gauge micro-incision sutureless vitrectomy with valved cannulas (Constellation Vision System, Alcon Laboratories, Inc, Forth Worth, TX, USA) under a sub-tenon's local anesthesia consisting of a mixture of xylocaine $2 \%$ and ropivacaine $0.8 \%$. Honan's balloon was not used in this case. The balanced salt solution (BSS) for infusion had $0.5 \mathrm{~mL}$ of $1 ; 1,000$ adrenaline, diluting it to $1 ; 1,000,000$. After 


\section{Case Reports in Ophthalmology}

\begin{tabular}{l|l}
\hline DOI: 10.1159/000510558 & $\begin{array}{l}\text { C } 2021 \text { The Author(s). Published by S. Karger AG, Basel } \\
\text { www.karger.com/cop }\end{array}$ \\
\hline
\end{tabular}

Stavrakas et al.: Paracentral Acute Middle Maculopathy following Surgically Induced Branch Retinal Artery Occlusion During Vitrectomy

confirmation of a posterior vitreous detachment, a core vitrectomy was carried out, without the use of adjuvant triamcinolone acetonide. The intraocular pressure (IOP) setting was switched on during surgery at a set pressure of $25 \mathrm{~mm} \mathrm{Hg}$. During core vitrectomy constant bleeding of an avulsed inferior temporal arteriole, relatively close to the optic disc, was recognized and was considered the source of the vitreous hemorrhage. As hemostasis was not achieved by IOP elevation to $80 \mathrm{~mm} \mathrm{Hg}$ for $2 \mathrm{~min}$, endodiathermy was used in order to ensure the clearance of the media and the continuation of the surgery. Peripheral vitrectomy and vitreous base shaving were then performed. Search of the periphery by means of scleral depression, without aspiration, failed to disclose any retinal breaks or holes or otherwise suspicious retinal areas. As a result, BSS was used to fill the vitreous cavity at the end of the surgery. Apart of the 2 min elevation of IOP in order to achieve hemostasis, in no other instance did the IOP surpass the set pressure of $25 \mathrm{~mm} \mathrm{Hg}$ throughout surgery. At the end of the surgery, a subconjunctival injection of cefuroxime and dexamethasone was administered.

Postoperatively, on day 1, and while BCVA of the affected eye had already improved to $20 / 100$, routine post-op follow-up examination revealed a subtle but sizable and well-demarcated retinal whitening involving the inferior macula. Upon questioning, the patient admitted the existence of a paracentral scotoma, corresponding to the fundoscopic finding. SD-OCT showed thickening and hyperreflectivity of both the inner and middle retinal layers, as seen in Figure 1. On the basis of this finding, a fluorescein angiography was carried out 1 week later in order to allow adequate media clearance. The fluorescein angiography identified a branch retinal artery occlusion, demonstrating delayed perfusion and patchy filling of an inferior retinal arteriole, starting at the site of the intraoperative endodiathermy application (Fig. 2). By that time, a diagnosis of a surgically induced branch retinal artery occlusion exhibiting both a cotton wool spot and a PAMM lesion and thus affecting both superficial and intermediate capillary plexuses, was made. Intraocular pressure at the time of diagnosis was $12 \mathrm{~mm} \mathrm{Hg}$.

Three months postoperatively and while BCVA has improved to 20/40, SD OCT demonstrates a remarkable thinning and atrophy of the inner and middle retinal layers and the patient continues to experience a stable paracentral scotoma (Fig. 2).

\section{Discussion/Conclusion}

In post-mortem studies in human donor eyes, Tan et al. identified four different capillary plexuses in the human retina: the first two located in superficial layers, i.e., the nerve fiber layer and the ganglion cell layer, thus referred to as the superficial capillary plexus, another located at the junction of the inner plexiform layer and superficial boundary of the inner nuclear layer (intermediate capillary plexus) and the last located at the junction of the deep inner nuclear and outer plexiform layer border (deep capillary plexus) [7]. Since its introduction, PAMM's underlying pathophysiological mechanism has been consistently hypothesized to be one of infarction of both the middle and inner retina representing the final stage of an ischemic cascade [8]. Our case affords an insight into those pathological processes involved providing angiographic evidence of the retinal ischemic changes responsible for PAMM development.

In our case, surgically induced branch retinal artery occlusion of a second order inferior temporal retinal artery resulted in the formation of a PAMM lesion in combination with ischemic manifestations deriving from the superficial capillary plexus in the form of a cotton wool spot, at as early as the first postoperative day. We speculate that the onset of PAMM and cotton wool spot formation was immediate but could not be detected during surgery since retinal whitening commences to appear 45-60 min after an ischemic event [9], by which time 
surgery was already over. Fluorescein angiography revealed a delayed filling of the occluded artery as well as a relative hypofluoresence at the site of the lesion, probably representing a masking effect. So, an angiographically proven branch retinal artery occlusion leads to a prompt formation of both a cotton wool spot and a PAMM lesion, located focally to the parafoveal area and not to the whole perfusion area, representing ischemia separately involving both superficial and intermediate capillary systems. The parafoveal localization of these lesions may be attributed to the greater density of the infarct-related artery's capillary network in the area. A combination of both superficial and intermediate capillary ischemia is proved to be the most common finding in retinal artery occlusion as shown by a retrospective observational study of 40 eyes with some form of retinal artery occlusion, with a percentage as high as $78 \%$ among them exhibiting fundoscopic findings deriving from both plexuses [2].

Admittedly, in this case, more than one factors could have contributed to retinal ischemia leading to PAMM development postoperatively. Apart from the obvious endodiathermy application and subsequent surgically induced branch retinal artery occlusion, the marked elevation of intraocular pressure during vitrectomy could also induce retinal circulatory insufficiency. Additionally, the type of local anesthesia used has been proposed to contribute to transient retinal ischemia, as Swamy et al. [10] have suggested that the anesthetic fluid may become trapped in the periocular connective tissue causing focal mechanical compression of the central retinal artery. Even ropivacaine's and adrenaline's vasoconstrictive effects have been accused of causing a temporary reduction of the central retinal artery's blood flow [11]. Moreover, according to Sugiura et al. [12], rapid scleral depression without concomitant aspiration can provoke more severe elevation of intraocular pressure during search of the periphery. Finally, pre-existing vasculopathies, as is systemic hypertension in our case, are considered a risk factor for PAMM [13, 14].

In conclusion, our case provides angiographic evidence of ischemia involving both superficial and intermediate capillary plexuses leading to a prompt formation of both cotton wool spot and PAMM lesions. Our aim is to raise awareness of this potential complication of vitrectomy surgery, especially when involving multiple traumatic manipulations onto the retinal surface, in addition to the impact of the nature of the surgery on the central retinal artery's blood flow itself. Many of the patients' subjective complaints may be overlooked, especially when visual acuity is preserved or even improved and is therefore of ophthalmologists to suspect and identify ischemic lesions deriving from deeper capillary plexuses and counsel patients accordingly.

\section{Statement of Ethics}

The patient consented to publication of the case in writing.

\section{Conflict of Interest Statement}

The authors have no conflicts of interest to declare.

\section{Funding Sources}

No funding or grant support was received for this case report. 


\section{Case Reports in Ophthalmology}

\section{Author Contributions}

All authors attest that they meet the current ICMJE criteria for authorship. Surgeon: P.S., ancillary testing: A.R., manuscript draft: A.A., E.K., P.T., K.A., and C.T.

\section{References}

1 Sarraf D, Rahimy E, Fawzi AA, Sohn E, Barbazetto I, Zacks DN, et al. Zacks DNet al. Paracentral acute middle maculopathy. JAMA Ophthalmol. 2013;131(10):1275.

2 Yu S, Pang CE, Gong Y, Freund KB, Yannuzzi LA, Rahimy E, et al. The spectrum of superficial and deep capillary ischemia in retinal artery occlusion. Am J Ophthalmol. 2015 Jan;159(1):53-63.e1.

3 Chen X, Rahimy E, Sergott RC, Nunes RP, Souza EC, Choudhry N, et al. Spectrum of Retinal Vascular Diseases Associated With Paracentral Acute Middle Maculopathy. Am J Ophthalmol. 2015 Jul;160(1):26-34.e1.

4 Hooshmand J, Moore P, McKay D, Vote BJ. Paracentral acute middle maculopathy associated with severe vision loss following vitrectomy for vitreous hemorrhage. Clin Exp Ophthalmol 2018; Jan 23. (E pub ahead of print)

5 Creese K, Ong D, Sandhu SS, Ware D, Alex Harper C, Al-Qureshi SH, et al. Paracentral acute middle maculopathy as a finding in patients with severe vision loss following phacoemulsification cataract surgery. Clin Exp Ophthalmol. 2017 Aug;45(6):598-605.

6 Nakashima H, Iwama Y, Tanioka K, Emi K. Paracentral Acute Middle Maculopathy following Vitrectomy for Proliferative Diabetic Retinopathy: Incidence, Risk Factors, and Clinical Characteristics. Ophthalmology. 2018 Dec;125(12):1929-36.

7 Tan PE, Yu PK, Balaratnsingam C, Cringle SJ, Morgan WH, McAllister IL, et al. Quantitative confocal imaging of the retinal microvasculature in the huma retina. Invest Ophthalmol Vis Sci. 2011;52(3):1486-92.

8 Bakhoum MF, Freund KB, Dolz-Marco R, Leong BC, Baumal CR, Duker JS, et al. Paracentral Acute Middle Maculopathy and the Ischemic Cascade Associated With Retinal Vascular Occlusion. Am J Ophthalmol. 2018 Nov;195:143-53.

9 Ashton N, Dollery CT, Henkind P, Ramalho PS, Hill DW. Focal retinal ischemia: ophthalmoscopic, circulatory and ultrastructural changes. Br J Ophthalmol. 1966;50:281-8.

10 Swamy BN, Merani R, Hunyor A. Central retinal artery occlusion after phacoemulsification. Retin Cases Brief Rep. 2010;4(3):281-3.

11 Findl O, Dallinger S, Menapace R, Rainer G, Georgopoulos M, Kiss B, et al. Effects of peribulbar anesthesia on ocular blood flow in patients undergoing cataract surgery. Am J Ophthalmol. 1999 Jun;127(6):645-9.

12 Sugiura Y, Okamoto F, Okamoto Y, Hiraoka T, Oshika T. Intraocular pressure fluctuation during microincision vitrectomy with constellation vision system. Am J Ophthalmol. 2013 Nov;156(5):941-947.e1.

13 Rahimy E, Kuehlewein L, Sadda SR, Sarraf D. Paracentral acute middle maculopathy. Retina. 2015 Oct;35(10):1921-30.

14 Nemiroff J, Kuehlewein L, Rahimy E, Tsui I, Doshi R, Gaudric A, et al. Assessing deep retinal capillary ischemia in paracentral acute middle maculopathy by optical coherence tomography angiography. Am J Ophthalmol. 2016 Feb;162:121-132.e1. 


\section{Case Reports in Ophthalmology}
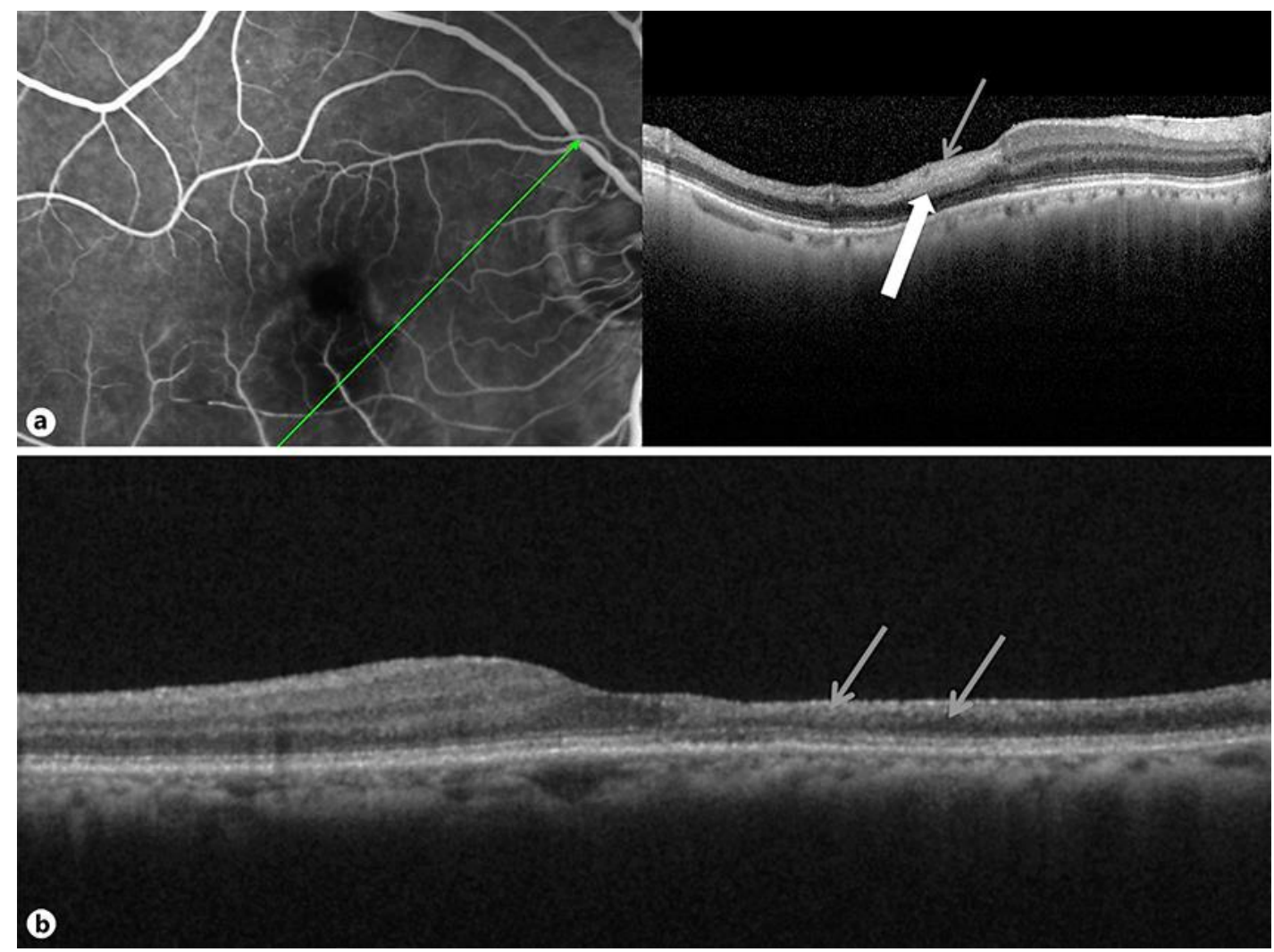

Fig. 1. a SD-OCT image depicting thickening and hyperreflectivity of both the inner (yellow arrow) and middle (white thick arrow) retinal layers. b SD-OCT image 3 months postoperatively. Notice the remarkable thinning and atrophy of the inner and middle retinal layers (yellow arrows). 


\section{Case Reports in Ophthalmology}

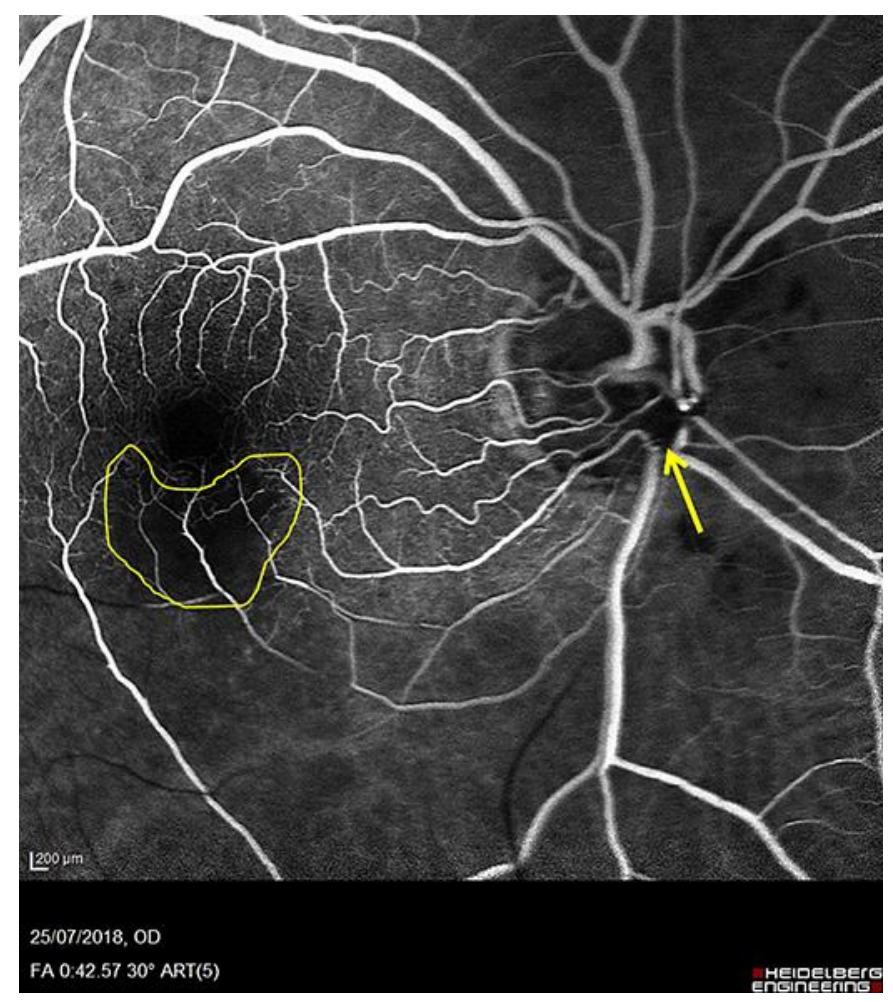

Fig. 2. Fluorescein angiography image depicting delayed filling of the occluded artery and the site of intraoperative endodiathermy deliverance (yellow arrow). The cotton wool spot and PAMM lesion localization giving rise to a relative masking effect is also depicted (yellow line).

Stavrakas et al.: Paracentral Acute Middle Maculopathy following Surgically Induced Branch Retinal Artery Occlusion During Vitrectomy 